\title{
Contents and Methods on Psychological Testing of College Students
}

\author{
Yanjun Luo \\ Students Safety and Life Service Center, Bohai University, Jinzhou, 121013, China \\ luoyanjun5885@163.com
}

Keywords: college students; psychological testing; contents and methods; mental health

\begin{abstract}
Chinese college students' mental health is worrying, psychological health has become the important influence factors of college students' individual development and school stability. This paper analyzes the importance of college students' mental health, illustrates the content of college students' psychological test and gives the method of college students' psychological test. In view of Intelligence test, it gives the methods of " Wechsler Intelligence scale, Raven standard Intelligence tests, Stanford - Binet Intelligence scale"; To Personality test, it gives the methods of "Minnesota multiphasic per - sonality inventory, Cartel the sixteen move factor questionnaire, Eysenck move questionnaire"; For Clinical evaluation test, it gives the "Self - reporting inventory, Self - rating 'scale, Self - rating anxiety scale" methods; And in view of the Vocational test, it gives the methods of "Vocational ability test tools, Strong - Campbell interest inventory, Hollander 's self - directed search".
\end{abstract}

\section{Introduction}

Mental health, is the important aspect of modern healthy inseparable, refers to all aspects of the psychological process and activity in the condition of a good or normal. The ideal state of mental health is to maintain correct perfect personality, normal intelligence, correct cognitive, proper emotion, reasonable will, positive and appropriate behavior and a good condition. Corresponding with the mental health is psychological sub-health and psychopathic. The standard definition of mental health: it has good adaptability; Can fully understand yourself and to your ability to make appropriate evaluation. The purpose of life practical; Not divorced from reality environment; To maintain the complete and harmonious personality; Good at learning from experience; To maintain a good interpersonal relationship; Can reasonably to vent their emotions and controlling their emotions; Without runs against the interests of the collective, can bring personality; On the premise of not against social norms, can properly meet the basic needs of individuals.

College students' psychological has many characteristics of mid youth, but as a special group, college students can't entirely equivalent and social youth. Academic problems, emotional problems, interpersonal relationship, anxiety, emotional problems, sexual health, psychological health problems of special group and college life adaptation is a common mental health problems of college students. Chinese college students' mental health is worrying, there is a considerable number of college students' negative psychological mood; Drop out of college students because of mental illness, to drop out of school for more than $50 \%$ of the total number of mental health has become the important factors that affect college students' individual development and school stability. To strengthen college students' mental health education is to promote the healthy growth of college students under the new situation, cultivating the important way of top creative talents, is to fully implement the party's education policy, the construction of the important measures of human resource power, is to promote higher education reform, strengthen and improve ideological and political education's important task. Through the study of the psychological test of college students, the establishment of mental health files, build perfect mental health prevention system and serve for the healthy growth of college students.

\section{Importance on Mental Health of College Students}

Early youth is the most valuable mental results found his inner world. College students with the 
in-depth development of self-awareness, self-esteem is more and more strong, but due to the choosing profession, inferiority and puppy love problems, many students have psychological problems. And these psychological problems seriously affected the students' learning work and normal life. The importance of college students' mental health embodied in the following several aspects [1, 2]:

(1) Psychological health is the foundation of university student. Mental health and a person's achievement, contribution and is of great talent, can promote college students to form healthy psychological quality, is the basic requirement of college students' all-round development, and to the society in the future, an intelligence in jobs, actively engaged in social activities, and continuously to a higher level of the development of the important conditions. Mental health can make college students overcome depend on psychology, enhance independence, develop healthy personality psychological and is college students' solid psychological foundation of success.

(2) Mental health is the need of comprehensive implementation of quality education. Quality education attaches great importance to the people's ideological and moral quality, ability, personality development, physical health and mental health education. Fully implementing quality education is to cultivate students' innovation spirit and practice ability, improving the students' psychological quality, make have ideal, morality, culture and discipline, moral, intellectual, physical all-round development of the socialist cause builders and successors. College students don't have good psychological quality, can't adapt to the needs of social development, it is difficult to undertake the task of the socialist cause, the psychological quality education is the starting point of quality education, is the important task of higher education, also need to strengthen college students psychological health education.

(3) Conducive to the healthy growth of college students' mental health. The individual mental characteristics of college students, often refers to the psychological and behavioral performance and steady of a variety of features, often characterized by two main temperament and personality. Temperament is mainly emotion reflects the characteristics of the character in addition to the characteristics of temperament contains, including will reflect the characteristics of the. The psychological characteristics of contemporary college students is generally characterized by being active, good at independent thinking, participation awareness is stronger, vigorous spirit and so on, which is conducive to the healthy growth of college students.

(4) Mental health directly affects the development level of personality. Students in the process of psychological health education to accept the code of ethics, the behavior way, environmental information and social expectations and gradually improve their own personality structure. The values of objective existence as a measure to their mental life, evaluation and regulation also affect the development of the main body personality. Mental health education is attached to this transformation is not negatively, but move to guide the educatees adjustment during the transformation direction, to know their own behavior evaluation, to achieve the goal of psychological optimization, healthy personality.

\section{Contents on Psychological Testing of College Students}

There are many contents of the psychological test, the main content of college students' psychological tests include the following:

(1) Intelligence test. The continuous development of society and economy, education can not only pay attention to imparting knowledge, also must pay attention to discover and develop Intelligence. College students' intelligence development and intellectual structure, not only directly affects the period of school learning, also affect the cost of the future and development. Intelligence test is related to people's normal mental function of the floorboard of the various tests that is the test of general ability. Modern psychologists have different opinions on intelligence. The so-called intelligence is refers to man's ability to learn and adapt to the environment. Intelligence includes observation ability, memory, imagination and thinking ability, etc. Intelligence directly affects the success of a person in the society. Test of intelligence is the intelligence science test, the main test a 
person's thinking ability, learning ability and the ability to adapt to the environment.

(2) Personality test. Personality refers to a person the sum of relatively stable psychological activity characteristic, is whether a person can use talents, the basis of effective work done. Personality is the unification of the individual mental characteristics, these characteristics determine the explicit behavior and implicit behavior of people, and make them have the stability of the difference with the behavior of others. Uniqueness and diversity, recessive, stability and unity are mostly defined accepted personality characteristics. Personality is made up of many aspects, including personality, interests, hobbies, temperament and values, etc. Personality test is also called the individuality test, characteristic measuring inclination of uniqueness and individual behavior. The role of personality test includes five aspects: personnel selection and human resources management, career planning, counseling and counseling, clinical psychological diagnosis and academic research.

(3) Clinical evaluation test. Clinical evaluation test in psychological counseling and therapy, psychiatry, neurology, and other clinical departments have widely application. Usually adopt evaluation questionnaire as a tool, it is the mental health workers engaged in research and clinical practice. Evaluation questionnaire has some of the characteristics of psychological test, but in form and difference. Evaluation questionnaire is through the comparison, the behavior of the individual can be compared and quantitative, and divided into several levels, this is the important basis for the development of rating scale. The role of the rating scale includes the following aspects: as objective tools for collecting data, to evaluate the mental health status and disease severity, grouped data as the research sample, evaluate the effect of psychological and drug intervention, observing the progress of the disease, assessment and related factors of mental health.

(4) Vocational test. It includes Vocational ability test and Vocational interest test. Vocational ability test is of a person's verbal ability, mathematical ability, spatial judgment ability, ability to observe details, writing ability, motor coordination ability, ability, social communication ability and organization and management ability to make a comprehensive evaluation [3]. Vocational ability test is for self-discovery, clear the characteristics of their own capabilities tools, also is enterprise or business the unit recruitment, selection, cultivating talents of all kinds of commonly used tools; Vocational interest test is a method of psychological test, show that a person is the most interested in and what is most likely to get satisfied job, can be used to personal interest and those who work in a more successful employee's interest in comparison, is to know a person's interest orientation and interest in a test sequence.

\section{Methods on Psychological Testing of College Students}

According to the content and the college students' psychological characteristics of college students' psychological test, the proposed method of psychological test is shown in Fig. 1.

\begin{tabular}{|c|c|c|c|}
\hline & \multicolumn{2}{|c|}{ Methods on psychological testing of college students } & \\
\hline \multirow[b]{2}{*}{ Intelligence test } & & & \multirow{2}{*}{ Vocational test } \\
\hline & Personality test & Clinical evaluation test & \\
\hline \begin{tabular}{|c|} 
Wechsler intelligence \\
scale \\
\end{tabular} & $\begin{array}{l}\text { Minnesota multiphasic } \\
\text { per-sonality inventory }\end{array}$ & $\begin{array}{l}\text { Self-reporting } \\
\text { inventory }\end{array}$ & $\begin{array}{l}\text { Vocational ability } \\
\text { test tools }\end{array}$ \\
\hline $\begin{array}{l}\text { Raven standard } \\
\text { intelligence tests }\end{array}$ & $\begin{array}{l}\text { Cartel the sixteen } \\
\text { personality factor } \\
\text { questionnaire }\end{array}$ & $\begin{array}{c}\text { Self-rating } \\
\text { depression scale }\end{array}$ & $\begin{array}{l}\text { Strong-Campbell } \\
\text { interest inventory }\end{array}$ \\
\hline $\begin{array}{c}\text { Stanford-Binet } \\
\text { intelligence scale }\end{array}$ & $\begin{array}{c}\text { Eysenck personality } \\
\text { questionnaire }\end{array}$ & $\begin{array}{l}\text { Self-rating anxiety } \\
\text { scale }\end{array}$ & $\begin{array}{c}\text { Hollander's } \\
\text { self-directed search }\end{array}$ \\
\hline
\end{tabular}

Fig. 1. Methods on psychological testing of college students 
(1) "Intelligence test" includes the following three ways: Wechsler Intelligence scale, scale and operation scale of two parts including words, provide total IQ scores, verbal IQ scores and IQ scores operation, not only can evaluate a person's general Intelligence can also learn about other different ability differences; Raven standard intelligence tests [4], designed to test the general intelligence level, especially to measure people's ability to solve problems, observation, thinking ability, find and use of the information they need and the ability to adapt to social life. The main characteristic is suitable for wide age range, test object does not limited by culture, race and language; Stanford - Binet intelligence scale [5], with age as measuring intelligence scale, provisions of a certain age should reach a certain level of intelligence. Test item grouping children by age, every age group of the test is composed of six projects, including painting, fold and give a definition of the word, judge the meaning and recall the story, from many aspects, such as reasoning activities with age increase, item difficulty also gradually increased.

(2) "Personality test" includes the following three methods [6-8]: Minnesota multiphasic per sonality inventory, including 10 clinical scale and four validity scale. Apply to at least 16 years old, has more than primary school culture level, have no effect on test results of physical defects, is psychological counseling workers and mental medical workers are one of the necessary psychological test. From several aspects to comprehensive investigation, the person's psychology is being used in the world's most one of personality test; Cartel the sixteen move factor questionnaire, the measurement of normal personality trait constructs is an integral part of Cattell 's comprehensive going of intrapersonal psychological variables covering individual differences in cognitive abilities, normal move traits, abnormal move traits, dynamic motivational traits, mood states, and transitory that states which are all seems into account in his behavioral specification prediction equation; Eysenck personality questionnaire, is medicine, justice, education, and counseling in areas such as one of the most widely used questionnaire. Has high reliability and validity, the measured results can be obtained at the same time a variety of experimental psychology research, and validation according to the theory of personality dimensions.

(3) "Clinical evaluation test" includes the following three methods: Self - reporting inventory [9], the world's most famous one of the mental health test scale, has a large capacity, rich reflect the symptoms, and more accurately depict the participants' subjective symptom, etc. Contains a broader learning content, psychotic symptoms in feeling, mood, thinking, behavior and living habits, interpersonal relationship, diet, sleep and so on have been involved; Self - rating 'scale [10], it is contains 20 items, divided into 4 rating scale of self-assessment, characteristic is easy to use, and can be quite visually reflect the subjective feeling of depression patients. Mainly applies to adults presenting with symptoms of depression, including outpatient and hospitalized patients; Self - rating anxiety scale, is a fairly simple clinical tools for analyzing patient subjective symptom, suitable for adults presenting with symptoms of anxiety, has wide applicability. It can better reflect the anxiety tendency of psychosis patients' subjective feeling.

(4) "Vocational test" includes two kinds of methods: the first is "Vocational ability test tools", is used to examine whether a person has a career in a certain potential, mainly used for the selection and employment. Including common profession ability test pilot, administrative vocational ability test, quality test teachers ability test, doctor ability test and secretarial ability test, etc. The second category is "vocational interest test", commonly used two methods: one is that the Strong Campbell interest inventory, is the most widely used in the employment guidance occupational interest survey. Is mainly an empirical questionnaires, rather than a strict scale, does not have a theoretical foundation and support of statistics. There are evaluation of a total of 420 questions and 10 professional groups. Man version can provide evaluation about 54 professional groups, women's version of the evaluation can provide about 36 professional team; Second, Hollander 's self directed search, from the perspective of interest to explore the problems of vocational guidance, vocational interest explicitly put forward the personality view, make people awareness of vocational interest there has been a qualitative change. As occupational interest survey tool, Holland tries to find out two matches for each career interests of vocational ability. The combination of the interest and ability of testing in the career guidance and career counseling played a significant role in 
practice.

\section{Conclusion}

College students' mental health education is a complicated systems engineering and engineering innovation. To strengthen college students' mental health education is to promote the healthy growth of college students under the new situation, cultivating the important way of top creative talents, is to fully implement the party's education policy, the construction of the important measures of human resource power, is to promote higher education reform, strengthen and improve ideological and political education's important task. Research results in this paper to determine the mental health problems of college students, to carry out mental health education and psychological consultation provide effective basis, for improving college students' psychological quality, promote the development of college students' physical and mental health, train qualified builders and successors for socialism plays an important role in such aspects.

\section{References}

[1] H. Di, "The role of psychological test in the psychological health education of college students," Education Teaching Forum, vol. 6, no. 4, pp. 269-270, 2014.

[2] G. Yang, "Psychological testing and its application in college students' psychological archives," Lantai World, vol. 30, no. 17, pp. 57-58, 2015.

[3] Mental growth site, "Professional ability test," http://types.yuzeli.com/survey/careerability, 2016-5-25.

[4] X. X. Fan, H. Zhong, L. F. Liu, et al., "Intelligence test and analysis of standard Raven medical students in tibet region," Health Vocational Education, vol. 34, no. 5, pp. 126-128, 2016.

[5] N. Wen, "Trial study on fourth edition for Stanford-Binet intelligence scale," Master's degree of East China Normal University, 2005.

[6] J. S. Che, "Application of psychological test in career planning of college students," Journal of Taiyuan Urban Vocational College, vol. 17, no. 3, pp. 59-62, 2015.

[7] X. Huang, "Review on methods of personality assessment at home and abroad," Journal of Henan Institute of Education (Natural Science Edition), vol. 22, no. 4, pp. 48-51, 2013.

[8] S. Y. Zhao, "A Facet Theory Study on Construct Validity of Eysenck Personality Questionnaire," Journal of Shandong Normal University (Humanities and Social Sciences), vol. 57, no. 4, pp. 146-150, 2012.

[9] S. L. Chen, X. P. Gao, L. J. Li, et al., "A preliminary evaluation of the reliability and validity of the PTSD symptom checklist," Chinese Mental Health Journal, vol. 19, no. 6, pp. 373-376, 2005.

[10] D. X. Zhang, J. H. Luo, L. Z. Peng, et al., "Factor Analysis on Survey Results of the Self Rating Depression Scale (SDS) in Students," Journal of Kunming Medical University, vol. 33, no. 5, pp. 61-63, 2012. 